Article

\title{
In-depth Analysis of the Lid Subunits Assembly Mechanism in Mammals
}

\author{
Minghui Bai, Xian Zhao, Kazutaka Sahara, Yuki Ohte, Yuko Hirano, Takeumi Kaneko, \\ Hideki Yashiroda and Shigeo Murata *
}

Laboratory of Protein Metabolism, Graduate School of Pharmaceutical Sciences, The University of Tokyo, 7-3-1 Hongo, Bunkyo-ku, Tokyo 113-0033, Japan; hakumeikei@gmail.com (M.B.); zhao-xian762@g.ecc.u-tokyo.ac.jp (X.Z.); kztkshr@gmail.com (K.S.); yuki.ohte@gmail.com (Y.O.); yuuko92130@yahoo.co.jp (Y.H.); takeumi@gmail.com (T.K.); yashiroda@mol.f.u-tokyo.ac.jp (H.Y.)

* Correspondence: smurata@mol.f.u-tokyo.ac.jp; Tel.: +81-3-5841-4803

Received: 25 April 2019; Accepted: 29 May 2019; Published: 31 May 2019

check for updates

\begin{abstract}
The 26S proteasome is a key player in the degradation of ubiquitinated proteins, comprising a $20 \mathrm{~S}$ core particle (CP) and a 19S regulatory particle (RP). The RP is further divided into base and lid subcomplexes, which are assembled independently from each other. We have previously demonstrated the assembly pathway of the $\mathrm{CP}$ and the base by observing assembly intermediates resulting from knockdowns of each proteasome subunit and the assembly chaperones. In this study, we examine the assembly pathway of the mammalian lid, which remains to be elucidated. We show that the lid assembly pathway is conserved between humans and yeast. The final step is the incorporation of Rpn12 into the assembly intermediate consisting of two modular complexes, Rpn3-7-15 and Rpn5-6-8-9-11, in both humans and yeast. Furthermore, we dissect the assembly pathways of the two modular complexes by the knockdown of each lid subunit.
\end{abstract}

Keywords: 26S proteasome; 19S regulatory particle; lid subcomplex; Rpn proteins; assembly

\section{Introduction}

Protein degradation exerted by the ubiquitin-proteasome system starts with the conjugation of ubiquitin chains to target proteins. Polyubiquitinated proteins are then recognized and captured by the $26 \mathrm{~S}$ proteasome and digested to short peptide fragments [1]. The ubiquitin-proteasome system is required for various cellular processes such as DNA damage repair, cell cycle progression, signal transduction, and immune response [2].

The 265 proteasome is made up of the $20 S$ core particle (CP) and the 195 regulatory particle (RP) [3]. As a prerequisite for protein degradation by the $\mathrm{CP}$, substrate proteins need to be recruited, deubiquitinated, and unfolded by the RP [4]. The RP can be further divided into a "base" and a "lid" subcomplex. The base subcomplex is composed of six ATPase subunits (Rpt1-Rpt6) and three non-ATPase subunits (Rpn1, Rpn2, and Rpn13), whereas the lid subcomplex is composed of nine non-ATPase subunits (Rpn3, Rpn5-Rpn9, Rpn11, Rpn12, and Rpn15/Sem1), and Rpn10 appears to mainly bind the lid subunits [5-8].

The assembly of the $26 \mathrm{~S}$ proteasome is highly complex because of the large number of subunits that require precise association to form an active complex. Previous studies have demonstrated assembly pathways of the $\mathrm{CP}$ and the base subcomplex. CP biogenesis, consisting of seven different $\alpha$-type subunits $(\alpha 1-\alpha 7)$ and seven different $\beta$-type subunits $(\beta 1-\beta 7)$, requires the assistance of at least five proteasome-specific chaperones called PAC1-4/Pba1-4 and POMP/Ump1 in mammals/yeast [9-16]. The assembly of the base subcomplex involves another set of chaperones, including p28/Nas6, p27/Nas2, 
S5b/Hsm5, and PAAF1/Rpn14 $[17,18]$. In both the CP and the base, the order of assembly has been clarified in detail using the siRNA-mediated knockdown of each subunit $[17,19]$.

Recent biochemical studies showed that the lid and the base form independently $[17,20]$. Studies using yeast mutants and the mass spectrometry analysis of native complexes suggested that the lid consists of two modules: a complex comprised of Rpn5, Rpn6, Rpn8, Rpn9, and Rpn11 and a complex comprised of Rpn3, Rpn7, and Rpn15/Sem1 (also called DSS1 in mammals; hereafter called Rpn15) [21-23]. These two modules seem to be connected via Rpn6 [22]. Rpn12 incorporation depends on the pre-assembly of all the other lid subunits and occurs as the last step of the lid assembly, which drives lid-base joining [24].

In addition, the molecular architecture of the $26 \mathrm{~S}$ proteasome was resolved in detail using cryoelectron microscopy, which revealed the subunit topology of the lid [25-27]. The PCI domain-containing subunits Rpn3, Rpn7, Rpn6, Rpn5, and Rpn9 form the fingers of the hand-shaped lid structure. The PCI domain is thought to mediate protein-protein interactions. Rpn8 connects Rpn3 and Rpn9, and Rpn11 lies in the palm of the hand. This structure is consistent and would explain the results regarding lid assembly which have been reported so far. However, the questions of how each subunit assembles into the modules and how the modules assemble into the lid remain to be answered.

In this study, we investigated the biogenesis of the mammalian lid subcomplex using a combination of RNA interference and mass spectrometry. siRNA knockdown of each lid subunit caused a characteristic change in distributions of the other lid subunits separated by glycerol gradient centrifugation. We purified complexes from the separated fractions as lid intermediates by immunoprecipitation. The components of the intermediates were determined by mass spectrometry. These analyses demonstrated the assembly pathway of the two modules of the lid subcomplex, as well as the assembly pathway of the lid in detail.

\section{Materials and Methods}

\subsection{Cell Culture and DNA Constructs}

HEK293T cells were cultured at $37^{\circ} \mathrm{C}$ with $5 \% \mathrm{CO}_{2}$ in Dulbecco's modified Eagle's medium (Nacalai Tesque, Kyoto, Japan) containing 10\% fetal bovine serum (Invitrogen, Waltham, Massachusetts, USA) and 1\% penicillin-streptomycin (Nacalai Tesque). The cDNAs encoding Rpn5, Rpn7, and Rpn9 were subcloned into the pIRESpuro3-Flag vector, and the cDNA encoding Rpn15 was subcloned into the pIRESpuro3-GFP vector. Transfection of these constructs into HEK293T cells was achieved by FuGENE 6 (Roche Applied Science, Penzberg, Germany), and the cells were selected with $4 \mu \mathrm{g} / \mathrm{mL}$ puromycin (InvivoGen, San Diego, California, USA).

\subsection{RNA Interference}

The siRNAs targeting human lid subunits (Table 1) were transfected into HEK293T cells using Lipofectamine RNAiMAX (Invitrogen) at a final concentration of $50 \mathrm{nM}$. For each sample, $9 \times 10^{5}$ cells were plated in a 10-cm dish $6 \mathrm{~h}$ before transfection, and the cells were harvested $48 \mathrm{~h}$ after transfection. 
Table 1. RNAi sequences used in this study on the assembly pathway of the lid subcomplex.

\begin{tabular}{ccc}
\hline Name & Sequence & Supplier \\
\hline Human Rpn3 & 5'-UGUCCUGACAGCUUGAGUCAGAAGG-3' & Invitrogen \\
Human Rpn5 & 5'-UUCCAUAGUCCUCAACAAGUGUGGA-3' & Invitrogen \\
Human Rpn6 & 5'-UAGUAAGUUAUCAUACAACUUGGCC-3' & Invitrogen \\
Human Rpn7 & 5'-UAGUAAGGAGCCAUGUUGUUAUCGC-3' & Invitrogen \\
Human Rpn8 & 5'-UGUGGUACCAGCCAACUAUUCUUUC-3' & Invitrogen \\
Human Rpn9 & 5'-AAUUGUUUCCUUUGUAACCUGUAGG-3' & Invitrogen \\
Human Rpn11 & 5'-AUACCAACCAACAACCAUCUCCGGC-3' & Invitrogen \\
Human Rpn12 & 5'-UAUGUCACGGGCCAGAAUUAGCUGC-3' & Greiner bio-one \\
Human Rpn15 & 5'-UAACAGACCUAAGUCUACCGGCUGC-3' & Greiner bio-one \\
\hline
\end{tabular}

\subsection{Protein Extraction, Immunological Analysis, and Antibodies}

Cells were lysed in lysis buffer [20 mM Tris- $\mathrm{HCl}$ ( $\mathrm{pH}$ 7.5), 0.2\% (w/w) NP-40, $1 \mathrm{mM}$ dithiothreitol, $2 \mathrm{mM}$ ATP, and $5 \mathrm{mM} \mathrm{MgCl} 2$ ] and incubated at $4^{\circ} \mathrm{C}$ for $10 \mathrm{~min}$. The lysates were retrieved by centrifugation at $20,000 \mathrm{~g}$ for $10 \mathrm{~min}$ at $4{ }^{\circ} \mathrm{C}$ and separated by $4 \%-24 \%$ glycerol gradient centrifugation as described previously $[19,28]$. Antibodies against human lid subunits, GFP, and Flag were used [17].

\subsection{Immunoprecipitation and Mass Spectrometry}

The accumulated intermediates were immunoprecipitated with M2 agarose (Sigma, St. Louis, Missouri, USA) as described previously [17]. The precipitated complexes were analyzed by LC-MALDI followed by tandem mass spectrometry using a TOF/TOF5800 analyzer (Applied Biosystems, Waltham, Massachusetts, USA).

\subsection{In Vitro Transcription and Translation}

In vitro labeling was performed using the TNT T7 Quick for PCR DNA system (Promega, Madison, Wisconsin, USA) with the EXPRESS Protein Labeling Mix $\left[{ }^{35} \mathrm{~S}\right]$ (PerkinElmer, Waltham, Massachusetts, USA), according to the manufacturer's protocol. The labeled samples were incubated with M2 agarose in a buffer containing $25 \mathrm{mM}$ Tris- $\mathrm{HCl}$ (pH 7.5), $150 \mathrm{mM} \mathrm{NaCl}$, and $0.2 \% \mathrm{NP}-40$. The washed beads were boiled in the SDS-sample buffer and separated by SDS-PAGE, followed by autoradiography visualizing.

\section{Results}

\subsection{Interdependence Between the Lid Subunits for Protein Expression}

To investigate the assembly pathway of the human lid subcomplex, we performed the knockdown of each lid subunit using siRNAs targeting Rpn3, Rpn5-Rpn9, Rpn11, Rpn12, and Rpn15 in HEK293T cells. The cells were harvested $48 \mathrm{~h}$ after siRNA transfection, when they were still viable. Further extension of any knockdown caused cell death, indicating that all the lid subunits are essential for viability in mammalian cells (data not shown). We examined the cell extracts by immunoblot analysis for each lid subunit, a base subunit Rpt6, and a CP subunit $\alpha 6$ (Figure 1A). Decreases in the targeted subunits were demonstrated, except for Rpn15, against which we failed to obtain an antibody (Figure 1A). The peptidase activity of the $26 \mathrm{~S}$ proteasome in each knockdown showed a $30-50 \%$ reduction compared with control cells, which also indicates successful knockdown of the lid subunits (Figure 1B).

The protein levels of Rpn3 and Rpn5-Rpn8 were decreased only by their corresponding siRNAs, and the protein levels of Rpn9, Rpn11, and Rpn12 were also decreased by siRNAs targeting other lid subunits. Rpn5-knockdown caused a reduction in Rpn9 and Rpn11 as well as Rpn5. Rpn11 was also decreased by Rpn6-knockdown and Rpn8-knockdown (Figure 1A). Because it has been suggested that these five subunits, i.e., Rpn5, Rpn6, Rpn8, Rpn9, and Rpn11, form a modular structure before the lid assembly, these results indicate interdependence for protein stability within this module. A 
severe reduction of Rpn12 was observed in Rpn3-knockdown, and a moderate reduction of Rpn12 was observed in Rpn7- and Rpn15-knockdown. Because Rpn3, Rpn7, and Rpn15 are also known to form a modular structure before the lid assembly, these results suggest that the protein expression of Rpn12 is dependent on the presence of the Rpn3-7-15 module. No decrease of the other subunits was observed in Rpn11- and Rpn12-knockdown, suggesting that these two subunits are not responsible for the stability or expression of the other subunits (Figure 1A).

A SiRNA

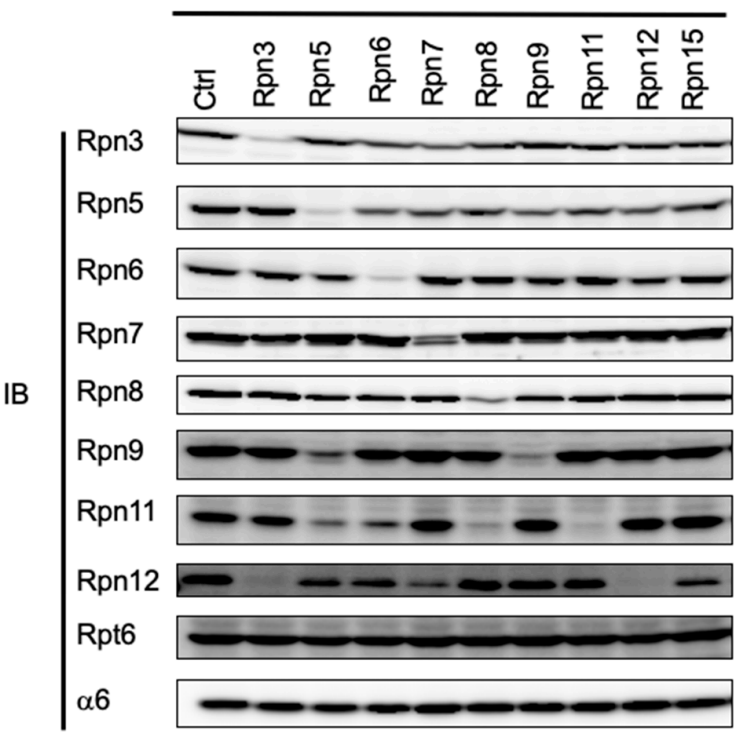

B

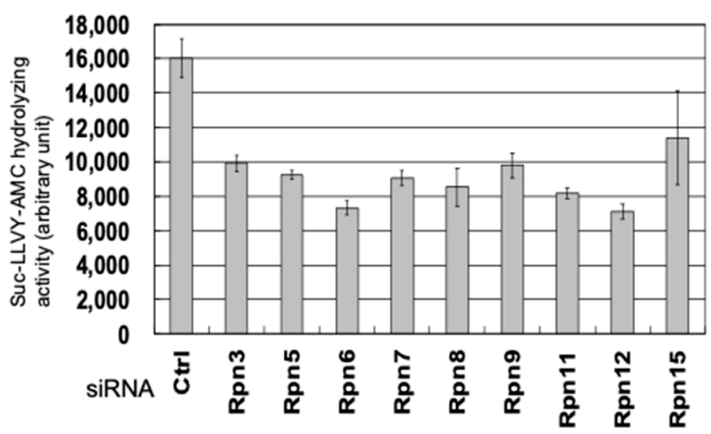

Figure 1. Interdependence between the lid subunits for protein expression. (A) siRNAs targeting Rpn3, Rpn5-Rpn9, Rpn11, Rpn12, and Rpn15 were transfected into HEK293T cells. The cell extracts $(20 \mu \mathrm{g})$ were then separated by native PAGE and were detected by immunoblot (IB) using antibodies against Rpn3, Rpn5-Rpn9, Rpn11, Rpn12, Rpt6, and $\alpha 6$. (B) The Suc-LLVY-AMC hydrolyzing activity of the knockdown of Rpn3, Rpn5-Rpn9, Rpn11, Rpn12, and Rpn15 was measured without added SDS (mean $\pm \mathrm{SD}, n=3$ ).

\subsection{Mammalian Cells Express a Lid-like Complex Without Rpn12 (LP2)}

We previously observed that mammalian cells contained an appreciable amount of complex containing lid subunits [17]. Lysates of HEK293T cells were separated by $4 \%-24 \%$ glycerol gradient centrifugation, followed by immunoblotting with antibodies for proteasome subunits (Figure 2A). Fraction 32 and Fraction 22 correspond to the peak location of the 26S proteasome (2.5 MDa) and the free CP (720 kDa), respectively, as indicated by the immunoblot analysis (Figure 2A). The $\alpha$-ring (280 kDa), composed of the seven $\alpha$-type subunits and PAC1-PAC2 and PAC3-PAC4 chaperone heterodimers, was also found around Fraction 12. In addition, all the lid subunits except Rpn12 had cosedimented in Fraction 12-14, suggesting that a subassembly of the lid exists in mammalian cells 
without any intervention (Figure 2A). Indeed, when we analyzed this complex by mass spectrometry utilizing HEK293T cells that expressed Rpn7-Flag, it turned out that this complex included all the lid subunits except Rpn12; however, we were not able to detect peptides derived from Rpn15, presumably because of the properties of the peptides (Figure 2B; see also Figure 5).

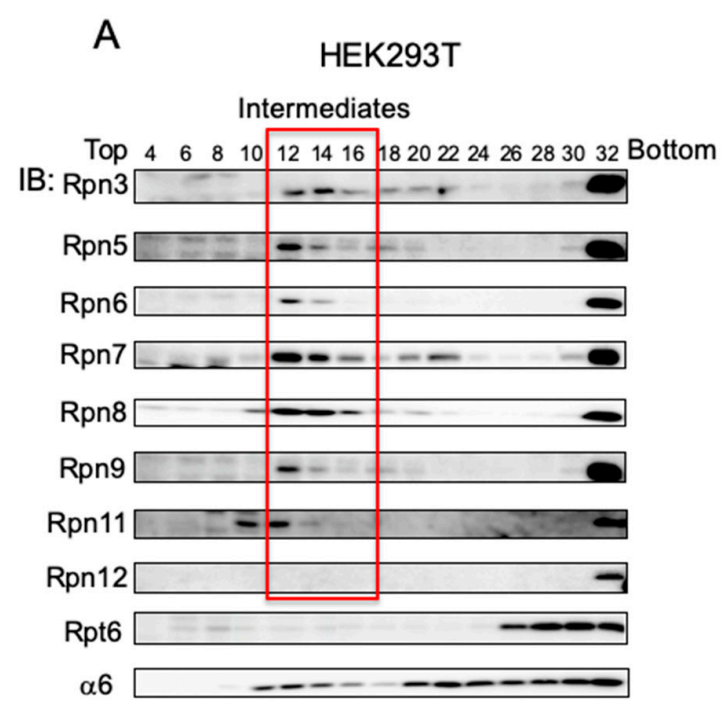

B

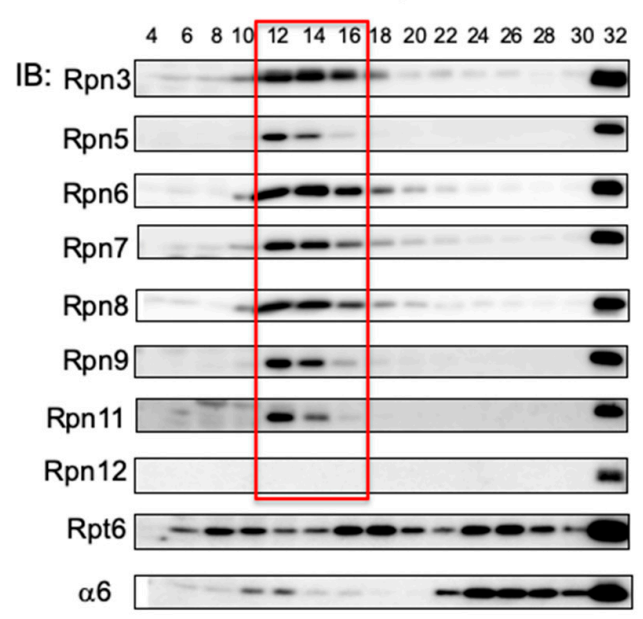

\begin{tabular}{|c|c|}
\cline { 2 - 2 } \multicolumn{1}{c|}{} & Cov(\%) \\
\cline { 2 - 2 } \multicolumn{1}{c|}{} & IP:Rpn7-Flag \\
\hline Rpn3 & 45.9 \\
\hline Rpn5 & 29.6 \\
\hline Rpn6 & 23.9 \\
\hline Rpn7 & 57.8 \\
\hline Rpn8 & 39.8 \\
\hline Rpn9 & 45.2 \\
\hline Rpn11 & 30.3 \\
\hline Rpn12 & \\
\hline
\end{tabular}

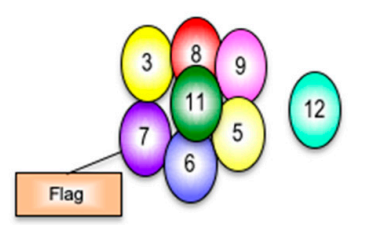

Figure 2. Rpn12 is incorporated at the final step of lid formation. (A) HEK293T cells stably expressing Rpn7-Flag were lysed and subjected to $4 \%-24 \%$ glycerol gradient centrifugation. The fractions were analyzed by immunoblotting with anti-Rpn3, Rpn5-Rpn9, Rpn11, Rpn12, Rpt6, and $\alpha 6$ antibodies. Fractions corresponding to the accumulated complexes (boxed in red) were immunoprecipitated (IP) with the anti-Flag antibody. The precipitated complexes were analyzed by LC-MALDI followed by tandem mass spectrometry. The table shows the sequence coverage of the identified subunits. (B) siRNA targeting Rpn12 was transfected into HEK293T cells for $48 \mathrm{~h}$ and then analyzed as described in Figure 2A. 
The same complex was reported as LP2 (lid particle 2) in yeast rpn12 mutants [24,29]. This complex was capable of completing lid assembly once Rpn12 had been added, and for subsequent assembly of the $26 \mathrm{~S}$ proteasome, showing that Rpn12 incorporation is the last step in the lid assembly of yeast [24]. Consistent with the observation in yeast, Rpn12-knockdown in HEK293T cells caused accumulation of the LP2 complex, which was further confirmed by mass spectrometry analysis of the complex (Figure 2B). At the same time, these cells accumulated aberrant complexes containing the base subunit Rpt6 in Fraction 8-10, Fraction 16-18, and Fraction 24-26, presumably corresponding to the Rpt3-Rpt6 module, the base subcomplex, and dimers of the base, respectively [17]. These results suggest that Rpn12 incorporation is the last step of lid assembly, after which the completed lid joins the base in mammalian cells.

\subsection{Rpn6 is Required for Interaction Between Rpn3-7-15 and Rpn5-8-9-11 and for Rpn11 Stability}

In the rpn6 yeast mutant, a complex comprised of Rpn5, Rpn8, Rpn9, and Rpn11 did accumulate, suggesting that this complex is connected with other subunits via Rpn6 [22]. When Rpn6 was knocked down in HEK293T cells, two intermediates appeared in Fraction 6-10 (Figure 3). One corresponded to a complex of Rpn3 and Rpn7 and the other to a complex of Rpn5, Rpn8, Rpn9, and Rpn11. This result indicates that the two complexes, Rpn3-7-15 and Rpn5-8-9-11, can be formed independently and are connected to each other through Rpn6, similar to the situation shown in yeast [22]. Interestingly, Rpn6-knockdown caused a reduction in Rpn11 (Figure 1A). Consistent with this, the relative abundance of Rpn11 seems to be lower than that of the LP2 complex observed in wild-type cells and Rpn12-knockdown cells, suggesting that the association of Rpn6 to the Rpn5-8-9-11 complex increases the stability of Rpn11 (Figure 3).
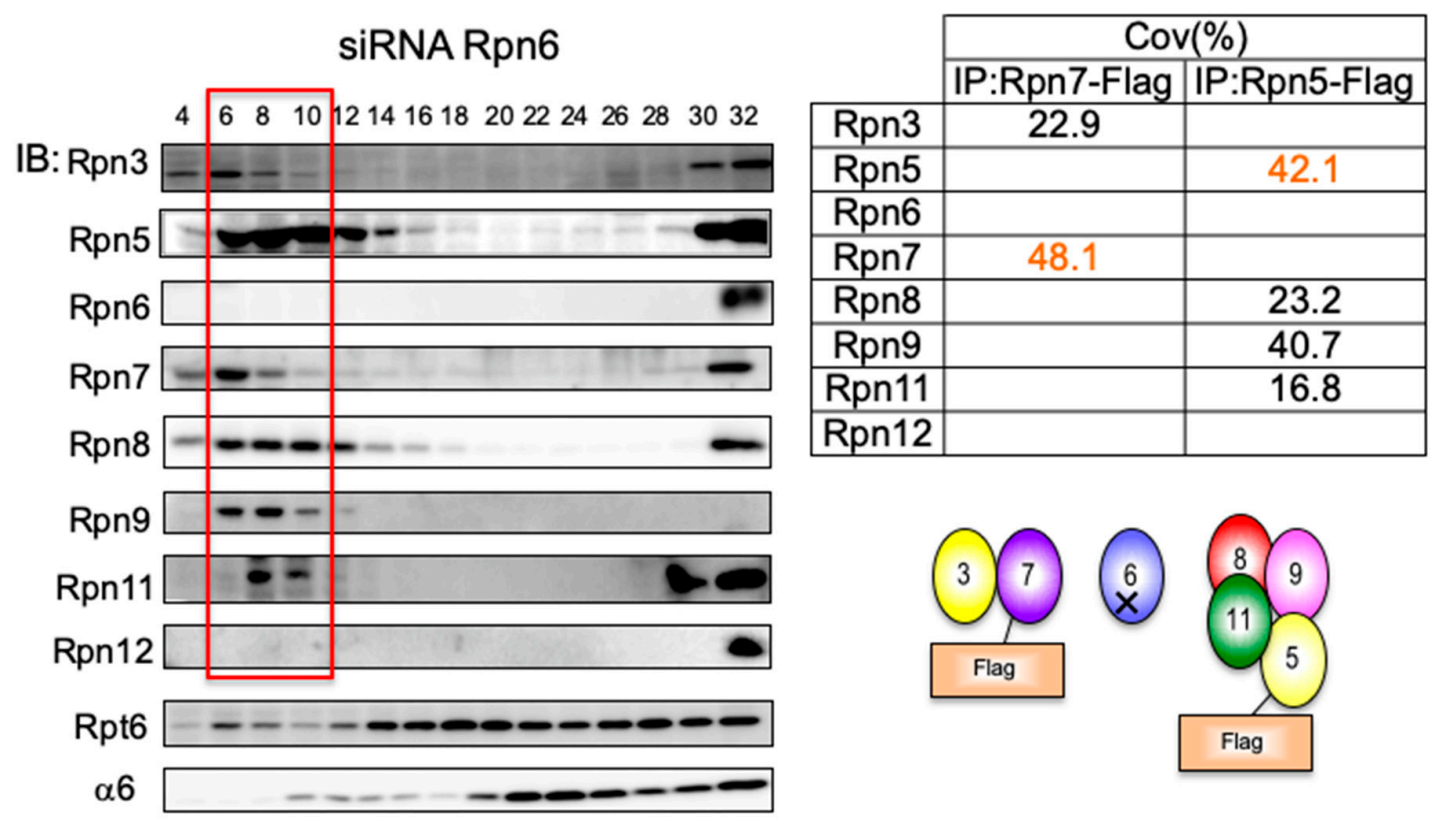

Figure 3. Rpn6 is required for the interaction between Rpn3-7-15 and Rpn5-8-9-11, and for Rpn11 stability. HEK293T cells stably expressing Rpn5-Flag and Rpn7-Flag treated with siRNA targeting Rpn6 for $48 \mathrm{~h}$ were analyzed in the same way as described in Figure 2. The table shows the sequence coverage of the identified subunits.

\subsection{Loss of Rpn11 does not Affect the Assembly of Other Lid Subunits but Affects Lid-base Joining}

Of the lid subunits, Rpn11 is the only subunit that has well-known catalytic activity, and its deubiquitinating activity is essential for the degradation of ubiquitinated proteins by the proteasome. To examine the role of Rpn11 in the lid assembly, knockdown of Rpn11 was performed. Unexpectedly, even without Rpn11, other lid subunits except Rpn12 were able to assemble into a complex (Figure 4, 
Fraction 10-14). However, the lid-base joining was severely impaired as indicated by the accumulation of the base subcomplex in Fraction 16-18 and Fraction 20-24 (Figure 4; blot for Rpt6). This finding is consistent with the notion that Rpn12 is a checkpoint that monitors the integrity of the LP2 complex and suggests that the association of Rpn11 is essential for lid-base joining, presumably by supporting proper conformation of the LP2 complex.

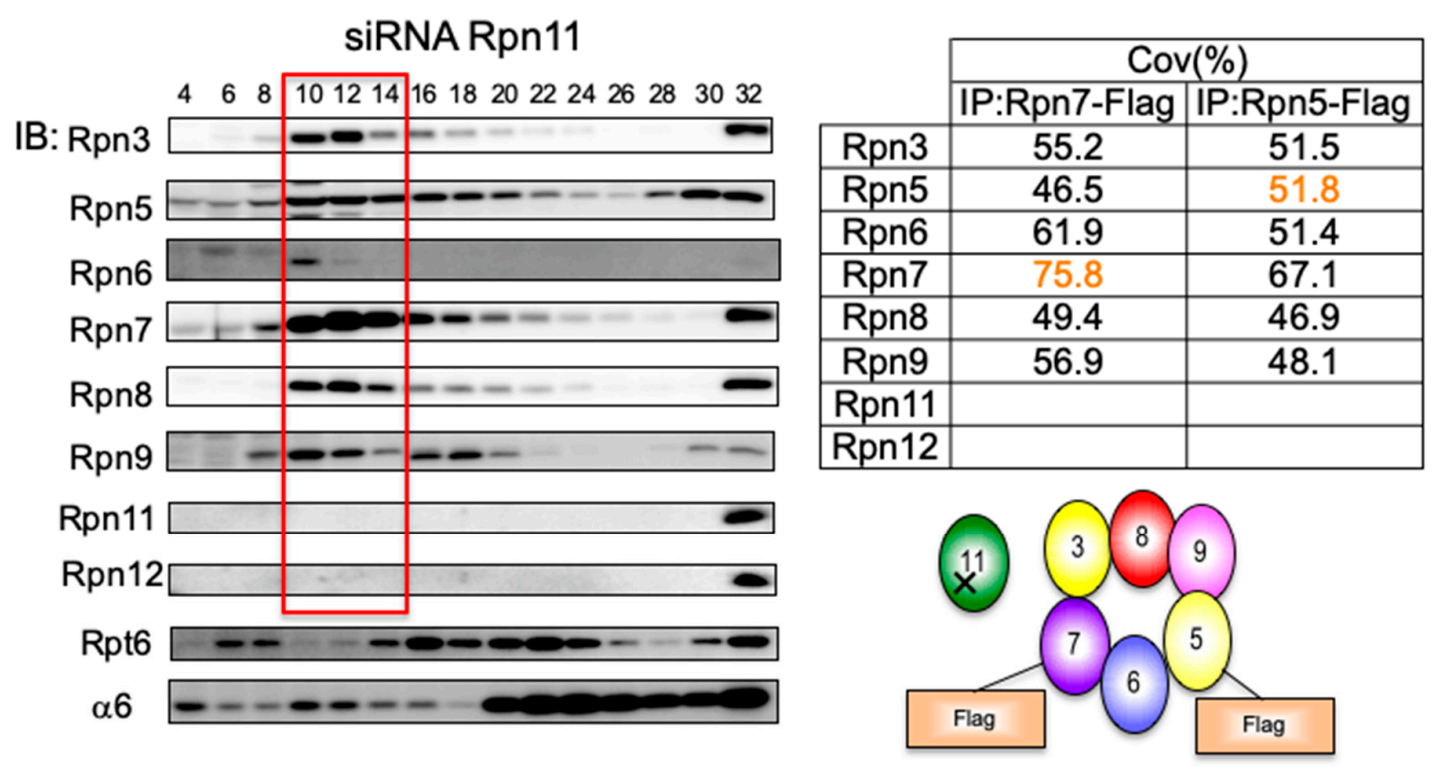

Figure 4. Loss of Rpn11 does not affect the assembly of other lid subunits but affects lid-base joining. HEK293T cells stably expressing Rpn5-Flag and Rpn7-Flag treated with siRNA targeting Rpn11 were analyzed for $48 \mathrm{~h}$ in the same way as described in Figure 2. The table shows the sequence coverage of the identified subunits.

\subsection{Interdependent Assembly of the Rpn5-8-9 Complex}

Rpn11-knockdown showed that Rpn11 is not an essential structural component in the formation of the Rpn5-8-9-11 complex. Therefore, we examined how the Rpn5-8-9 complex is assembled (Figure 5).

In Rpn5-, Rpn8-, or Rpn9-knockdown, the assembly of the Rpn3-7-15 module was not affected, and a small amount of Rpn6 was associated with the module, in favor of the Rpn3-7-15 module being assembled independently of the Rpn5-8-9-11 complex (Figure 5A-C). In Rpn5-knockdown, Rpn9 did not associate with Rpn8, as indicated by immunoprecipitation by Rpn9-Flag from the accumulated intermediates in Fraction 4-10 and mass spectrometry analysis of the resultant precipitates (Figure 5A). Through similar experiments, we found that Rpn5 did not associate with Rpn9 without Rpn8 (Figure 5B) and that Rpn5 did not associate with Rpn8 without Rpn9 (Figure 5C). These results indicate that Rpn5, Rpn8, and Rpn9 form a complex only when all the three subunits are present. This Rpn5-8-9 complex might serve as a core complex for the addition of the essential deubiquitylase Rpn11, because the loss of Rpn5 and Rpn8 significantly reduced the protein level of Rpn11 (Figure 1A). 
A

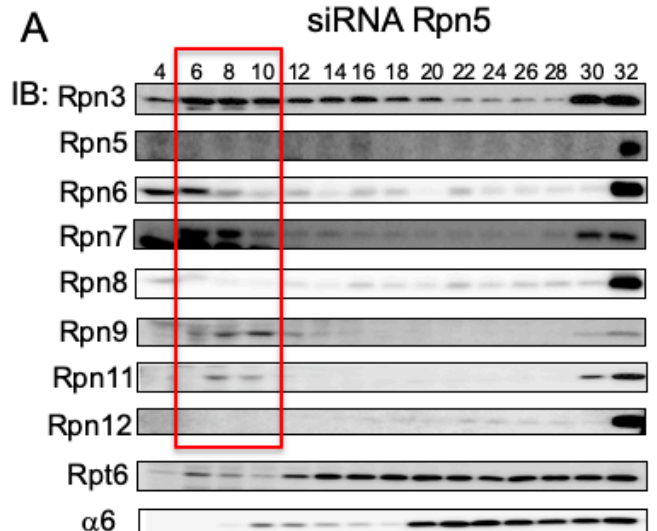

B

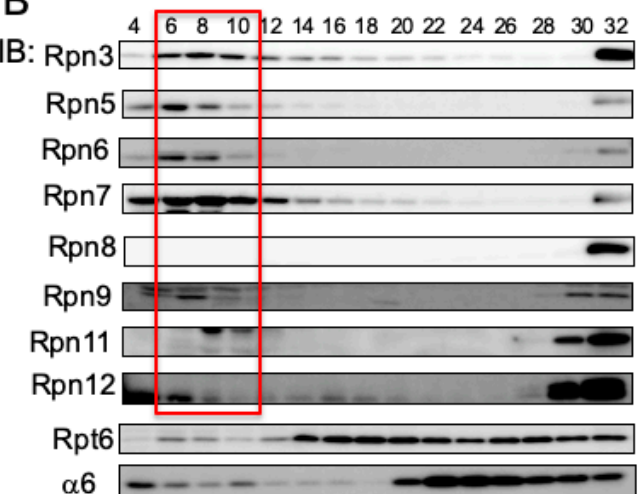

C

$\alpha 6-$

siRNA Rpn9

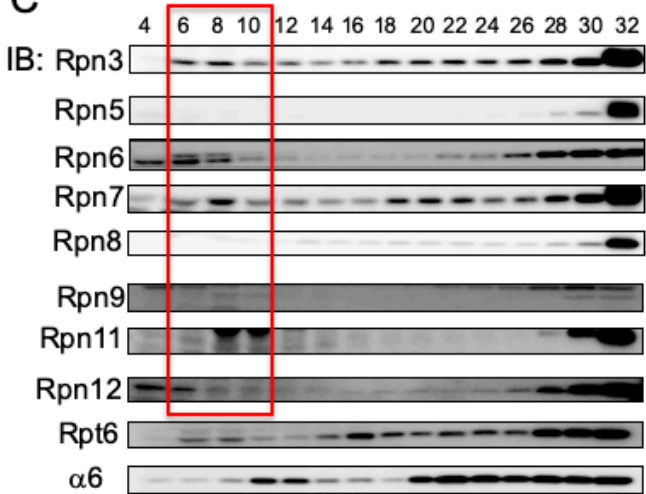

\begin{tabular}{|c|c|c|}
\hline & Cov & (\%) \\
\hline Rnn3 & IP:Rpn7-Flag & IP:Rpn9-Flag \\
\hline Rpn5 & & \\
\hline Rpn6 & 5.2 & \\
\hline Rpn7 & 51.7 & \\
\hline Rpn8 & & \\
\hline Rpn9 & & 29.3 \\
\hline Rpn11 & & \\
\hline
\end{tabular}

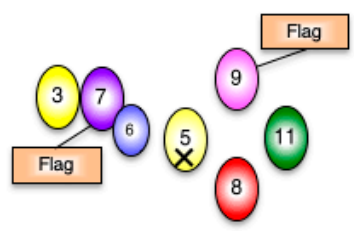

\begin{tabular}{|c|c|c|}
\cline { 2 - 3 } \multicolumn{1}{c|}{} & \multicolumn{2}{c|}{$\operatorname{Cov}(\%)$} \\
\cline { 2 - 3 } \multicolumn{1}{c|}{} & IP:Rpn7-Flag & IP:Rpn5-Flag \\
\hline Rpn3 & 32.6 & \\
\hline Rpn5 & & 13.8 \\
\hline Rpn6 & 9.2 & \\
\hline Rpn7 & 33.9 & \\
\hline Rpn8 & & \\
\hline Rpn9 & & \\
\hline Rpn11 & & \\
\hline Rpn12 & & \\
\hline
\end{tabular}

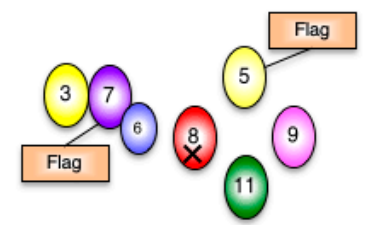

\begin{tabular}{|c|c|c|}
\cline { 2 - 3 } \multicolumn{1}{c|}{} & \multicolumn{2}{c|}{$\operatorname{Cov}(\%)$} \\
\cline { 2 - 3 } \multicolumn{1}{c|}{} & IP:Rpn7-Flag & IP:Rpn5-Flag \\
\hline Rpn3 & 38.8 & \\
\hline Rpn5 & 4.4 & 20.8 \\
\hline Rpn6 & 10.9 & \\
\hline Rpn7 & 56.8 & \\
\hline Rpn8 & & \\
\hline Rpn9 & & \\
\hline Rpn11 & & \\
\hline Rpn12 & & \\
\hline
\end{tabular}

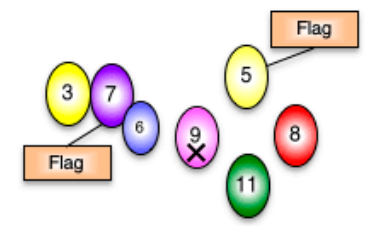

Figure 5. Interdependent assembly of the Rpn5-8-9 complex. HEK293T cells stably expressing Rpn7-Flag and Rpn9-Flag treated with siRNA targeting Rpn5 (A), cells stably expressing Rpn5-Flag and Rpn7-Flag treated with siRNA targeting Rpn8 (B), and cells stably expressing Rpn5-Flag and Rpn7-Flag treated with siRNA targeting Rpn9 (C) were analyzed in the same way as described in Figure 2. The tables show the sequence coverage of the identified subunits.

\subsection{Rpn7-Rpn6 Interaction Connects the Rpn3-7-15 Module with the Rpn5-6-8-9-11 Module}

The Rpn3-7-15 module associates with the Rpn5-8-9-11 complex via Rpn6 (Figure 3). We next investigated how the Rpn3-7-15 module is assembled and how it binds to Rpn6.

When Rpn3 was knocked down, Rpn7-Flag in the intermediates in Fraction 10-14 coprecipitated with the Rpn5-6-8-9-11 module (Figure 6A). In contrast, without Rpn7, Rpn3-Flag in the intermediate fraction did not associate with the Rpn5-6-8-9-11 module, which was readily formed in the absence of Rpn7 (Figure 6B). When immunoprecipitated with Rpn5-Flag in Rpn7-knockdown cells, a small amount 
of Rpn3 was detected, suggesting that Rpn3 could directly associate with the Rpn5-6-8-9-11 module although Rpn7 is required for efficient incorporation of Rpn3 to the lid. Rpn15-knockdown exhibited essentially the same phenotype as Rpn3-knockdown; a complex was accumulated that consisted of Rpn7 and the Rpn5-6-8-9-11 module and contained only a small amount of Rpn3 (Figure 6C). Although Rpn5 was readily detected by mass spectrometry analysis of the complex in Rpn15-knockdown cells, it was not detected by immunoblot analysis owing to a potential protein modification (Figure $6 \mathrm{C}$ ). These results suggest that the Rpn3-7-15 module interacts with Rpn6 via Rpn7, which connects the Rpn3-7-15 module with the Rpn5-6-8-9-11 module as a result. These data also suggest that Rpn15 is required for Rpn3 association with Rpn7.
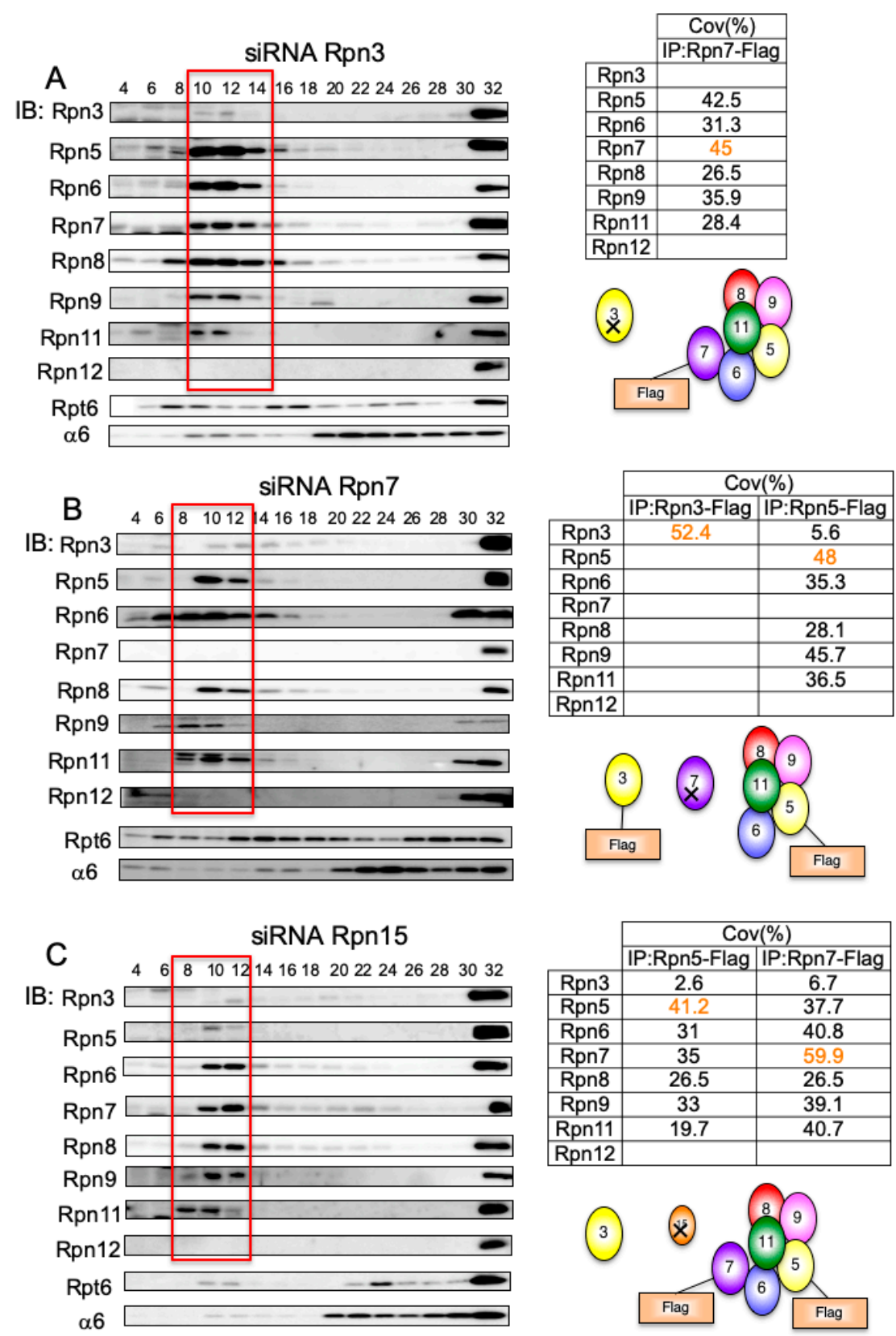

Figure 6. The Rpn7-Rpn6 interaction connects the Rpn3-7-15 module with the Rpn5-6-8-9-11 module. HEK293T cells stably expressing Rpn7-Flag treated with siRNA targeting Rpn3 (A), cells stably expressing Rpn3-Flag and Rpn5-Flag treated with siRNA targeting Rpn7 (B), and cells stably expressing Rpn5-Flag and Rpn7-Flag treated with siRNA targeting Rpn15 (C) were analyzed in the same way as described in Figure 2. The tables show the sequence coverage of the identified subunits. 


\subsection{Rpn15 Mediates the Association of Rpn3 with Rpn7}

As we were unable to identify endogenous Rpn15 either by mass spectrometry or by immunoblot analysis, we generated HEK293T cells stably expressing Rpn15-GFP to investigate the role of Rpn15 in the assembly of the lid subcomplex.

Knockdowns of Rpn7, Rpn3, and Rpn6 were performed in the Rpn15-GFP-expressing cells, and the cell lysates were fractionated by glycerol gradient centrifugation. Immunoblot analysis of each fraction essentially showed the same results as knockdown in normal HEK293T cells (data not shown; refer to Figure 2A, Figure 3 and Figure 6A,B). The normal LP2 complex of control cells (corresponding to Fraction 12-16 of Figure 2A), accumulated lid intermediates of knockdown cells (corresponding to Fraction 6-14 of Figure 3 and Figure 6A,B; referred to as "Lid" in Figure 7), and $26 \mathrm{~S}$ proteasomes (corresponding to Fraction 32 of Figure 2A, Figure 3 and Figure 6A,B; referred to as "26S" in Figure 7) of knockdown or control cells were immunoprecipitated with anti-GFP antibody.
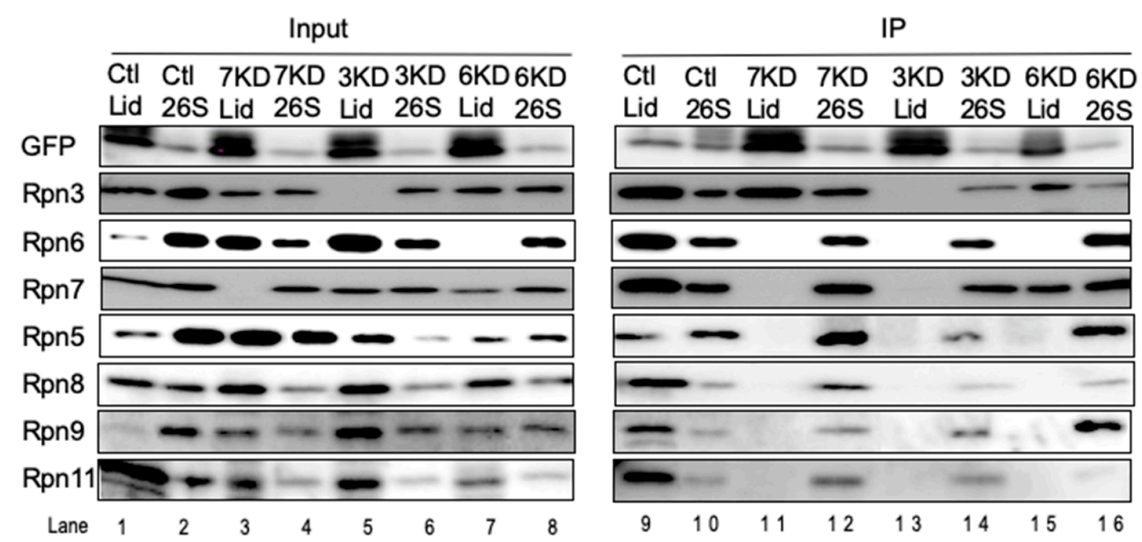

Figure 7. Rpn15 mediates the association of Rpn3 with Rpn7. siRNA targeting Rpn7, Rpn3, and Rpn6 was performed using HEK293T cells stably expressing Rpn15-GFP. Accumulated intermediates were collected and immunoprecipitated with anti-GFP antibody. When Rpn7 was knocked down, only Rpn3 was coimmunoprecipitated with Rpn15. None of the lid subunits was identified when Rpn3 was knocked down. Intermediates of Rpn3-7-15 were detected when Rpn6 was knocked down.

Subunits comprising the LP2 complex were coprecipitated from control lid fractions as well as 26S fractions with Rpn15-GFP, indicating that Rpn15 is a component of the LP2 complex (Figure 7, lanes 9, 10, 12, 14, and 16). When Rpn6 was knocked down, Rpn15 was coprecipitated with Rpn3 and Rpn7 but not with the Rpn5-6-8-9-11 module, confirming that Rpn15 is indeed a component of the Rpn3-7-15 module. However, Rpn15 associated only with Rpn3 in the absence of Rpn7 (Figure 7, lane 11) and did not associate with any other subunits in the absence of Rpn3 (Figure 7, lane 13). These results suggest that (i) the assembly of the Rnp3-7-15 module starts with Rpn3 and Rpn15 forming a complex and (ii) Rpn7 subsequent recruitment is dependent on Rpn15.

\subsection{Rpn15 Directly Binds to Rpn3 and Promotes Rpn3-Rpn7 Association in Vitro}

To further clarify how the Rpn3-7-15 module is assembled, Flag-Rpn3, Rpn7, and GFP-Rpn15 were cotranscribed/cotranslated in vitro in various combinations (Figure 8). Flag-Rpn3 pulled down GFP-Rpn15 in the absence of Rpn7 and did not pull down Rpn7 without Rpn15. This result indicates that Rpn3 directly interacts with Rpn15 and that Rpn3 and Rpn7 either do not interact or exhibit very weak interaction with each other (Figure 8A). However, Flag-Rpn3 pulled down Rpn7 in the presence of GFP-Rpn15, indicating that Rpn15 is required for the association between Rpn3 and Rpn7. 

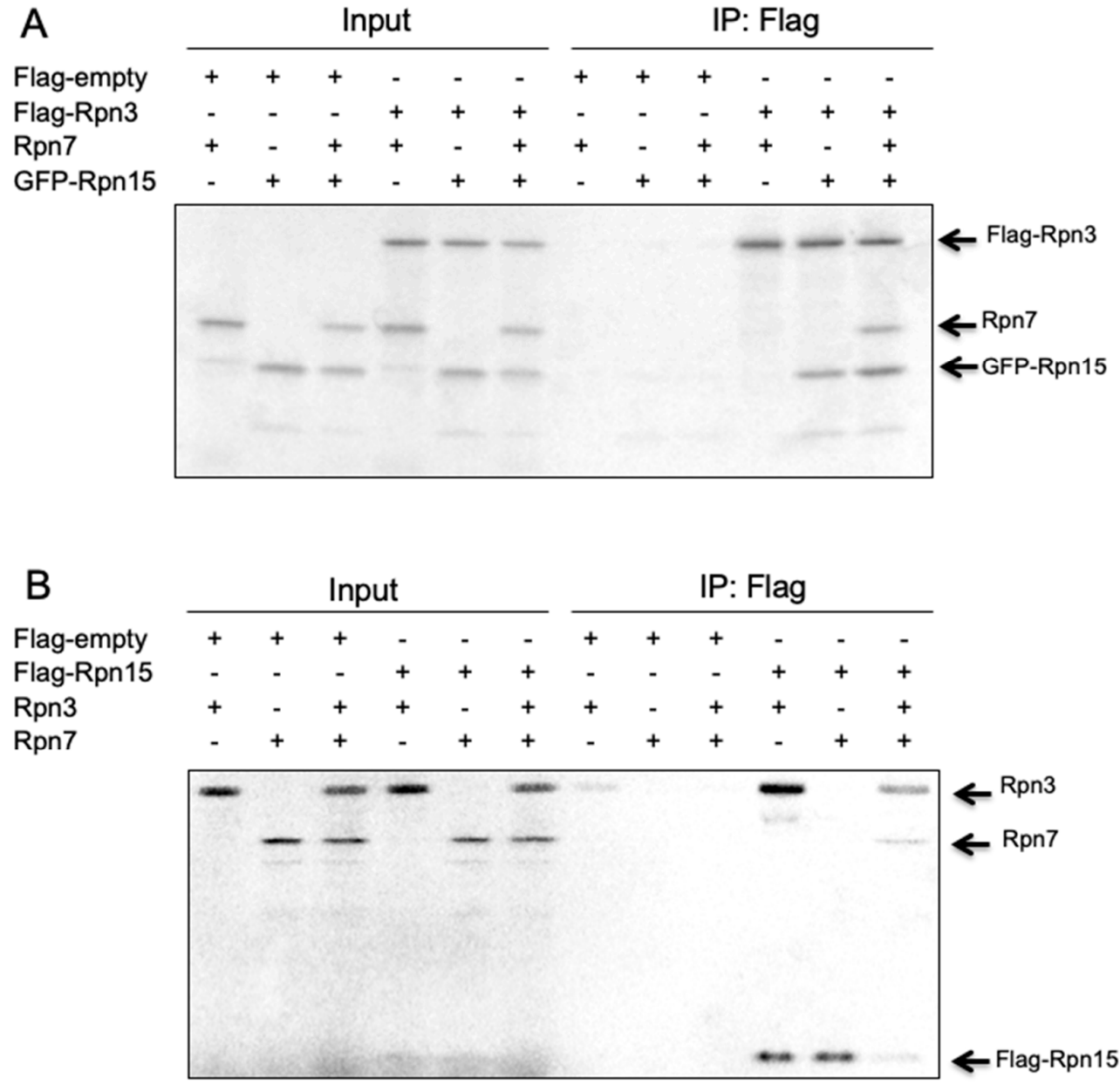

Figure 8. Rpn15 directly binds to Rpn3 and promotes Rpn3-Rpn7 association in vitro. Flag-Rpn3, Rpn7, and GFP-Rpn15 (A) or Flag-Rpn15, Rpn3, and Rpn7 (B) were cotranscribed/cotranslated and ${ }^{35}$ S-radiolabeled in reticulocyte lysates in various combinations as indicated. Anti-Flag agarose beads were added to the reaction mixture, and the immunoprecipitates were run on an SDS-PAGE gel. Coimmunoprecipitated proteins with Flag-Rpn3 (A) or Flag-Rpn15 (B) were visualized using autoradiography.

Similar results were observed by mixing Rpn3, Rpn7, and Flag-Rpn15 (Figure 8B). Flag-Rpn15 directly interacted with Rpn3 and pulled down Rpn7 only when Rpn3 was present. The binding of Rpn15 to Rpn3 might change the Rpn3 and/or Rpn15 conformation so that Rpn3 and/or Rpn15 can interact with Rpn7.

\section{Discussion}

Previous studies from yeast Saccharomyces cerevisiae have demonstrated that the assembly of the proteasome lid begins with the formation of two modular complexes: Rpn3-7-15 and Rpn5-6-8-9-11 [24,29,30]. These two modules are assembled, followed by the incorporation of Rpn12 that completes the lid assembly and drives lid-base joining.

In this study, we investigated the mammalian lid assembly pathway by observing complexes resulted from siRNA knockdown of each lid subunit and how the two modular complexes are formed. Although we cannot exclude the possibility that the intermediates we purified by immunoprecipitation are non-physiological intermediates such as those with abnormal subunit stoichiometries and dead-end products, we can describe the mammalian assembly pathway of the lid that conforms to the yeast model (Figure 9). Formation of the Rpn3-7-15 module starts with the association of Rpn15 and Rpn3, which enables Rpn7 incorporation. The Rpn5-6-8-9-11 module can be further divided into a Rpn5-8-9 
complex, Rpn6, and Rpn11 on the basis of interdependence between the subunits. Whereas the rpn11-1 mutation inhibits formation of the Rpn5-6-8-9-11 module and leads to accumulation of the Rpn3-7-15 module in S. cerevisiae [24], knockdown of Rpn11 in human cells indicates that mammalian Rpn11 is not required for the stability of the other lid subunits and can be incorporated even at the last step of LP2 formation. The minimal core of this module seems to be Rpn5-8-9, which can accommodate Rpn11. The association of Rpn6 to the core increases the capacity to accommodate Rpn11. The Rpn3-7-15 module and the Rpn5-6-8-9-11 module are connected between Rpn6 and Rpn7, forming the LP2 complex. Finally, Rpn12 is incorporated to complete lid assembly.

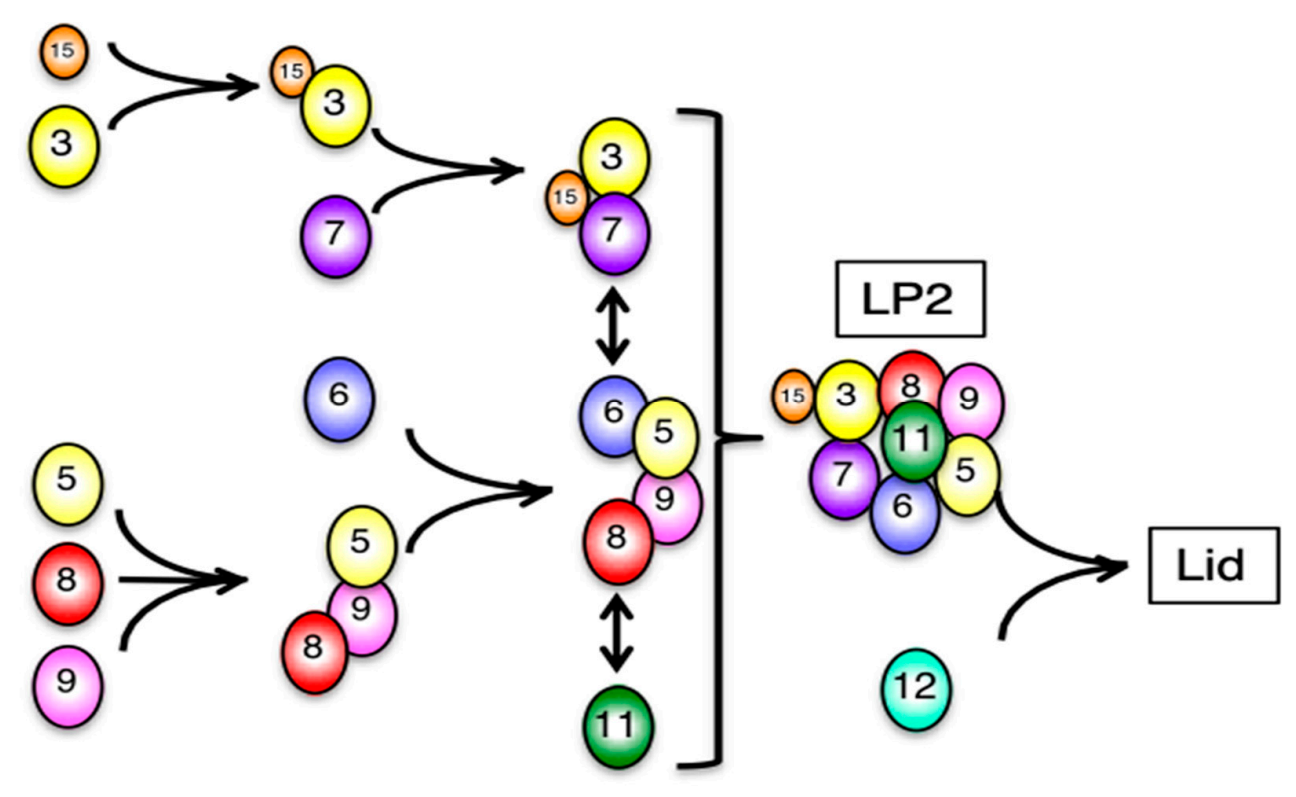

Figure 9. Assembly pathway of the mammalian proteasome lid subcomplex. The assembly of the lid subcomplex starts with the formation of two independent intermediates: Rpn3-15 and Rpn5-8-9. Then, Rpn7 and Rpn6 are incorporated into Rpn3-15 and Rpn5-8-9, forming Rpn3-7-15 and Rpn5-6-8-9, respectively. The Rpn5-6-8-9 complex is a prerequisite for incorporation of Rpn11. Rpn12 is the last subunit to be incorporated.

In our experiments, Rpn15 directly bound only to Rpn3, as reported previously [31]. Rpn15 did not bind to Rpn7 when Rpn3 was absent, and Rpn7 and Rpn3 did not interact with each other without Rpn15. Thus, we speculate that Rpn15 might interact first with Rpn3, which leads to potential conformational change in Rpn15 and/or Rpn3 to assist the incorporation of Rpn7 to the intermediate of Rpn3-7-15. On the other hand, it is reported that Sem1, yeast ortholog of Rpn15, can bind to both Rpn3 and Rpn7 independently via its N- and C-termini, and tethers together Rpn3 and Rpn7 [32]. Furthermore, Sem1 and Rpn15 are positioned between Rpn7 and Rpn3 in the cryoelectron microscopy structures of the $26 \mathrm{~S}$ proteasome [27,33]. Considering these preceding studies, it seems reasonable to suppose that Rpn15 also interacts with Rpn7 after binding to Rpn3, although our results do not necessarily require the direct interaction of Rpn15 to Rpn7.

As for another interesting issue regarding Rpn15, less accumulation of the base subunit Rpt6 was observed in Rpn15-knockdown cells than other lid subunits (Figure 6C). Despite the abnormality in the formation of the lid, accumulation of the base was also affected by the knockdown of Rpn15, which needs to be further clarified not only for the assembly mechanism but also at the transcriptional level.

Recent research shows that overexpression of the Rpn6 subunit in Caenorhabditis elegans and of Rpn11 in the fruit fly prolongs their lifespan [34]. In addition, the FOXO4-mediated upregulation of Rpn6 is a prerequisite for maintaining pluripotency in embryonic stem cells [35]. The overexpression of Rpn11 or Rpn7 is involved in DNA damage response [36,37]. However, the mechanisms are still 
unidentified, and elucidating the assembly pathway of the lid subcomplex under these circumstances with overexpressed lid subunits might help in understanding the underlying mechanisms.

Whether specific chaperones are involved in the mammalian lid assembly remains uncertain. We detected some proteins along with proteasome subunits in our mass spectrometry analysis (data not shown). We have not yet been able to discriminate whether these proteins are merely contaminated sediments or have potential biological importance. Although the lid can be reconstituted in Escherichia coli only with the expression of the yeast lid subunits [26,32], it may be possible to identify lid assembly chaperones among these proteins in future. Finally, the lid and the base, which are both assembled independently, are associated with each other to form the complete 19S RP. Further studies are needed to clarify the mechanism and the timing of the lid-base association.

\section{Conclusions}

This study describes the assembly pathway of the mammalian lid subcomplex of the RP in the 26S proteasome. The lid formation consists of the assemblies of two modular complexes: Rpn3-7-15 and Rpn5-6-8-9-11. The binding of Rpn15 to Rpn3 enables Rpn3 to interact with Rpn7. In Rpn5-6-8-9-11 formation, Rpn5-8-9 serves as a platform for the association of Rpn6 and Rpn11. Although Rpn11 binds to Rpn5-8-9 without Rpn6, Rpn6 has an effect on Rpn11 stability. The interaction between Rpn6 and Rpn7 puts Rpn3-7-15 and Rpn5-6-8-9-11 together. Rpn12 is the last subunit to be incorporated into the lid intermediate comprising all the other lid subunits, known as LP2.

Author Contributions: Conceptualization, M.B. and S.M.; methodology, M.B., K.S., Y.H., T.K. and S.M.; validation, X.Z., K.S., Y.O. and T.K..; investigation, M.B., X.Z., K.S., Y.O. and T.K.; writing—original draft preparation, M.B., K.S., H.Y. and S.M.; writing-review and editing, H.Y. and S.M.; visualization, M.B. and K.S.; supervision, S.M.; project administration, S.M.; funding acquisition, S.M.

Funding: This work was supported by JSPS KAKENHI Grant Numbers JP18H05500, JP18H04022, JP25221102, AMED Grant Number JP18gm1110003 AMED-CREST.

Conflicts of Interest: The authors declare no conflict of interest. The funders had no role in the design of the study; in the collection, analyses, or interpretation of data; in the writing of the manuscript, or in the decision to publish the results.

\section{References}

1. Baumeister, W.; Walz, J.; Zuhl, F.; Seemuller, E. The proteasome: Paradigm of a self-compartmentalizing protease. Cell 1998, 92, 367-380. [CrossRef]

2. Hershko, A.; Ciechanover, A. The ubiquitin system. Annu. Rev. Biochem. 1998, 67, 425-479. [CrossRef] [PubMed]

3. Glickman, M.H.; Ciechanover, A. The ubiquitin-proteasome proteolytic pathway: Destruction for the sake of construction. Physiol. Rev. 2002, 82, 373-428. [CrossRef] [PubMed]

4. Kohler, A.; Cascio, P.; Leggett, D.S.; Woo, K.M.; Goldberg, A.L.; Finley, D. The axial channel of the proteasome core particle is gated by the Rpt2 ATPase and controls both substrate entry and product release. Mol. Cell 2001, 7, 1143-1152. [CrossRef]

5. Saeki, Y.; Sone, T.; Toh-e, A.; Yokosawa, H. Identification of ubiquitin-like protein-binding subunits of the 26S proteasome. Biochem. Biophys. Res. Commun. 2002, 296, 813-819. [CrossRef]

6. Deveraux, Q.; Ustrell, V.; Pickart, C.; Rechsteiner, M. A 26 S protease subunit that binds ubiquitin conjugates. J. Biol. Chem. 1994, 269, 7059-7061.

7. Husnjak, K.; Elsasser, S.; Zhang, N.; Chen, X.; Randles, L.; Shi, Y.; Hofmann, K.; Walters, K.J.; Finley, D.; Dikic, I. Proteasome subunit Rpn13 is a novel ubiquitin receptor. Nature 2008, 453, 481-488. [CrossRef]

8. Rosenzweig, R.; Bronner, V.; Zhang, D.; Fushman, D.; Glickman, M.H. Rpn1 and Rpn2 coordinate ubiquitin processing factors at proteasome. J. Biol. Chem. 2012, 287, 14659-14671. [CrossRef]

9. Hirano, Y.; Hendil, K.B.; Yashiroda, H.; Iemura, S.; Nagane, R.; Hioki, Y.; Natsume, T.; Tanaka, K.; Murata, S. A heterodimeric complex that promotes the assembly of mammalian $20 S$ proteasomes. Nature 2005, 437, 1381-1385. [CrossRef] 
10. Hirano, Y.; Hayashi, H.; Iemura, S.; Hendil, K.B.; Niwa, S.; Kishimoto, T.; Kasahara, M.; Natsume, T.; Tanaka, K.; Murata, S. Cooperation of multiple chaperones required for the assembly of mammalian $20 \mathrm{~S}$ proteasomes. Mol. Cell 2006, 24, 977-984. [CrossRef]

11. Murata, S. Multiple chaperone-assisted formation of mammalian 20 S proteasomes. IUBMB Life 2006, 58, 344-348. [CrossRef] [PubMed]

12. Li, X.; Kusmierczyk, A.R.; Wong, P.; Emili, A.; Hochstrasser, M. beta-Subunit appendages promote $20 \mathrm{~S}$ proteasome assembly by overcoming an Ump1-dependent checkpoint. EMBO J. 2007, 26, 2339-2349. [CrossRef] [PubMed]

13. Le Tallec, B.; Barrault, M.B.; Courbeyrette, R.; Guerois, R.; Marsolier-Kergoat, M.C.; Peyroche, A. 20S proteasome assembly is orchestrated by two distinct pairs of chaperones in yeast and in mammals. Mol. Cell 2007, 27, 660-674. [CrossRef] [PubMed]

14. Yashiroda, H.; Mizushima, T.; Okamoto, K.; Kameyama, T.; Hayashi, H.; Kishimoto, T.; Niwa, S.; Kasahara, M.; Kurimoto, E.; Sakata, E.; et al. Crystal structure of a chaperone complex that contributes to the assembly of yeast $20 S$ proteasomes. Nat. Struct. Mol. Biol. 2008, 15, 228-236. [CrossRef] [PubMed]

15. Kusmierczyk, A.R.; Kunjappu, M.J.; Funakoshi, M.; Hochstrasser, M. A multimeric assembly factor controls the formation of alternative $20 S$ proteasomes. Nat. Struct. Mol. Biol. 2008, 15, 237-244. [CrossRef] [PubMed]

16. Wu, W.; Sahara, K.; Hirayama, S.; Zhao, X.; Watanabe, A.; Hamazaki, J.; Yashiroda, H.; Murata, S. PAC1-PAC2 proteasome assembly chaperone retains the core alpha4-alpha7 assembly intermediates in the cytoplasm. Genes Cells 2018, 23, 839-848. [CrossRef] [PubMed]

17. Kaneko, T.; Hamazaki, J.; Iemura, S.; Sasaki, K.; Furuyama, K.; Natsume, T.; Tanaka, K.; Murata, S. Assembly pathway of the Mammalian proteasome base subcomplex is mediated by multiple specific chaperones. Cell 2009, 137, 914-925. [CrossRef]

18. Funakoshi, M.; Tomko, R.J., Jr.; Kobayashi, H.; Hochstrasser, M. Multiple assembly chaperones govern biogenesis of the proteasome regulatory particle base. Cell 2009, 137, 887-899. [CrossRef]

19. Hirano, Y.; Kaneko, T.; Okamoto, K.; Bai, M.; Yashiroda, H.; Furuyama, K.; Kato, K.; Tanaka, K.; Murata, S. Dissecting beta-ring assembly pathway of the mammalian $20 \mathrm{~S}$ proteasome. EMBO J. 2008, 27, 2204-2213. [CrossRef]

20. Isono, E.; Nishihara, K.; Saeki, Y.; Yashiroda, H.; Kamata, N.; Ge, L.; Ueda, T.; Kikuchi, Y.; Tanaka, K.; Nakano, A.; et al. The assembly pathway of the $19 \mathrm{~S}$ regulatory particle of the yeast 26S proteasome. Mol. Biol Cell 2007, 18, 569-580. [CrossRef]

21. Isono, E.; Saeki, Y.; Yokosawa, H.; Toh-e, A. Rpn7 Is required for the structural integrity of the $26 \mathrm{~S}$ proteasome of Saccharomyces cerevisiae. J. Biol. Chem. 2004, 279, 27168-27176. [CrossRef] [PubMed]

22. Isono, E.; Saito, N.; Kamata, N.; Saeki, Y.; Toh, E.A. Functional analysis of Rpn6p, a lid component of the $26 \mathrm{~S}$ proteasome, using temperature-sensitive rpn6 mutants of the yeast Saccharomyces cerevisiae. J. Biol. Chem. 2005, 280, 6537-6547. [CrossRef] [PubMed]

23. Sharon, M.; Taverner, T.; Ambroggio, X.I.; Deshaies, R.J.; Robinson, C.V. Structural organization of the $19 S$ proteasome lid: Insights from MS of intact complexes. PLoS Biol. 2006, 4, e267. [CrossRef] [PubMed]

24. Tomko, R.J., Jr.; Hochstrasser, M. Incorporation of the Rpn12 subunit couples completion of proteasome regulatory particle lid assembly to lid-base joining. Mol. Cell 2011, 44, 907-917. [CrossRef] [PubMed]

25. Huang, X.; Luan, B.; Wu, J.; Shi, Y. An atomic structure of the human $26 \mathrm{~S}$ proteasome. Nat. Struct. Mol. Biol. 2016, 23, 778-785. [CrossRef] [PubMed]

26. Lander, G.C.; Estrin, E.; Matyskiela, M.E.; Bashore, C.; Nogales, E.; Martin, A. Complete subunit architecture of the proteasome regulatory particle. Nature 2012, 482, 186-191. [CrossRef]

27. Schweitzer, A.; Aufderheide, A.; Rudack, T.; Beck, F.; Pfeifer, G.; Plitzko, J.M.; Sakata, E.; Schulten, K.; Forster, F.; Baumeister, W. Structure of the human $26 \mathrm{~S}$ proteasome at a resolution of 3.9 A. Proc. Nat. Acad. Sci. USA 2016, 113, 7816-7821. [CrossRef]

28. Murata, S.; Udono, H.; Tanahashi, N.; Hamada, N.; Watanabe, K.; Adachi, K.; Yamano, T.; Yui, K.; Kobayashi, N.; Kasahara, M.; et al. Immunoproteasome assembly and antigen presentation in mice lacking both PA28alpha and PA28beta. EMBO J. 2001, 20, 5898-5907. [CrossRef]

29. Fukunaga, K.; Kudo, T.; Toh-e, A.; Tanaka, K.; Saeki, Y. Dissection of the assembly pathway of the proteasome lid in Saccharomyces cerevisiae. Biochem. Biophys. Res. Commun. 2010, 396, 1048-1053. [CrossRef]

30. Budenholzer, L.; Cheng, C.L.; Li, Y.; Hochstrasser, M. Proteasome Structure and Assembly. J. Mol. Biol. 2017, 429, 3500-3524. [CrossRef] 
31. Wei, S.J.; Williams, J.G.; Dang, H.; Darden, T.A.; Betz, B.L.; Humble, M.M.; Chang, F.M.; Trempus, C.S.; Johnson, K.; Cannon, R.E.; et al. Identification of a specific motif of the DSS1 protein required for proteasome interaction and p53 protein degradation. J. Mol. Biol. 2008, 383, 693-712. [CrossRef] [PubMed]

32. Tomko, R.J., Jr.; Hochstrasser, M. The intrinsically disordered Sem1 protein functions as a molecular tether during proteasome lid biogenesis. Mol. Cell 2014, 53, 433-443. [CrossRef] [PubMed]

33. Bohn, S.; Sakata, E.; Beck, F.; Pathare, G.R.; Schnitger, J.; Nagy, I.; Baumeister, W.; Forster, F. Localization of the regulatory particle subunit Sem 1 in the $26 S$ proteasome. Biochem. Biophys. Res. Commun. 2013, 435, 250-254. [CrossRef] [PubMed]

34. Vilchez, D.; Morantte, I.; Liu, Z.; Douglas, P.M.; Merkwirth, C.; Rodrigues, A.P.; Manning, G.; Dillin, A. RPN-6 determines C. elegans longevity under proteotoxic stress conditions. Nature 2012, 489, 263-268. [CrossRef] [PubMed]

35. Vilchez, D.; Boyer, L.; Morantte, I.; Lutz, M.; Merkwirth, C.; Joyce, D.; Spencer, B.; Page, L.; Masliah, E.; Berggren, W.T.; et al. Increased proteasome activity in human embryonic stem cells is regulated by PSMD11. Nature 2012, 489, 304-308. [CrossRef] [PubMed]

36. Tsolou, A.; Nelson, G.; Trachana, V.; Chondrogianni, N.; Saretzki, G.; von Zglinicki, T.; Gonos, E.S. The 19S proteasome subunit Rpn7 stabilizes DNA damage foci upon genotoxic insult. IUBMB life 2012, 64, 432-442. [CrossRef] [PubMed]

37. Butler, L.R.; Densham, R.M.; Jia, J.; Garvin, A.J.; Stone, H.R.; Shah, V.; Weekes, D.; Festy, F.; Beesley, J.; Morris, J.R. The proteasomal de-ubiquitinating enzyme POH1 promotes the double-strand DNA break response. EMBO J. 2012, 31, 3918-3934. [CrossRef]

(C) 2019 by the authors. Licensee MDPI, Basel, Switzerland. This article is an open access article distributed under the terms and conditions of the Creative Commons Attribution (CC BY) license (http://creativecommons.org/licenses/by/4.0/). 